\title{
Erhebliche Kluft
}

\author{
Die Suche nach sinnvollen Indikatoren für nachhaltige Entwicklung steht weiter- \\ hin auf der entwicklungs- und umweltpolitischen Agenda. Die momentanen \\ Trends gehen jedoch dahin, nachhaltige Entwicklung immer mehr mit Nachhaltig- \\ keit gleichzusetzen. Daher bedarf es einer Wende in der Indikatorenforschung, \\ um Grundbedürfnisbefriedigung wieder als integralen Bestandteil nachhaltiger \\ Entwicklung zu begreifen.
}

\begin{abstract}
S Berichts 1987 besteht zwar ein weitgehender Konsens in Wissenschaft und Politik, daß nachhaltige Entwicklung das Leitbild für Industrie- und Entwicklungsländer sein sollte. Tatsächlich besteht jedoch immer noch beträchtliche Konfusion darüber, was der Begriff nachhaltige Entwicklung konkret beinhaltet und wie er operationalisiert werden soll. Die Commission for Sustainable Development (CSD) fordert daher einen verbindlichen Indikatorenset zur Messung nachhaltiger Entwicklung bis zum Jahr 2000 (1).
\end{abstract}

\section{Ein mögliches Maß}

Eine mögliche Meßlatte für Nachhaltigkeit wurde von Pearce und Atkinson bereits 1993 aufgestellt (2). Nach ihrer Definition ist eine Ökonomie dann als nachhaltig zu bezeichnen, wenn der volkswirtschaftliche Kapitalstock über die Zeit mindestens konstant bleibt. Dies entspricht der Forderung aus dem Brundtland-Bericht, zukünftigen Generationen dieselbe Möglichkeit zur Bedürfnisbefriedigung $\mathrm{zu}$ geben wie der gegenwärtigen Generation. Entgegen der neoklassischen Wachstumstheorie verstehen Pearce/Atkinson jedoch volkswirtschaftliches Kapital nicht nur als Sachkapital, sondern als die Summe von Sach-, Human- und Naturkapital. Eine solche Klassifizierung wird mittlerweile auch von der Weltbank unterstïtzt. Da alle drei Kapitalformen Abschreibungen erfahren können, müssen somit die Nettoinvestitionen (d.h. Bruttoinvestitionen minus Abschreibungen aller drei Kapitalformen) größergleich Null sein, um den Gesamtkapitalstock zumindest konstant $\mathrm{zu}$ halten. Im Gleichgewicht muß daher auch die sogenannte ,echte“ Ersparnis (Ersparnis minus der Abschreibungen des Human-, Sach- und Naturkapitals) größergleich Null sein, um diese Inve- stitionen zu finanzieren. Bei dieser Operationalisierung wird folglich davon ausgegangen, daß alle drei Kapitalformen grundsätzlich substituierbar sind, d.h. eine Abnahme natürlichen Kapitals durch eine Zunahme von Sachkapital kompensiert werden kann. Eine solche Annahme ist natürlich weitgehend unrealistisch. Schließlich läßt sich eine Abnahme der Ozonschicht schwerlich durch die Zunahme irgendeiner Form von Sachkapital kompensieren. Daher wird eine solche Nachhaltigkeitsdefinition in der Literatur als „,schwache“ oder ,ökonomische“ Nachhaltigkeit definiert. Pearce/Atkinson plädieren zwar theoretisch für eine „kritische“ Nachhaltigkeitsdefinition, die den Erhalt eines nichtsubstituierbaren natïrlichen Kapitalstocks, wie z.B. bestimmter biologischer Arten verlangen würde (3).

In der Praxis ist die Identifizierung eines solchen kritischen Kapitalstocks jedoch problematisch. Schließlich ist das menschliche Wissen über das Funktionieren des Ökosystems nach wie vor sehr begrenzt. Daher erscheint die Operationalisierung einer „schwachen“ Nachhaltigkeitsdefinition mittels einer Berechnung der „echten“ Ersparnis momentan die einzig gangbare Alternative, um zumindest zu einer „Daumenregel“ für Nachhaltigkeit $\mathrm{zu}$ gelangen. Hamilton hat demonstriert, daß die empirische Anwendung der echten Ersparnis dramatische Resultate liefert (4). So zeichnet sich die Ländergruppe Sub-Sahara-Afrika in den achtziger Jahren zwar durch eine schwach positive „konventionelle“ Ersparnis (ohne Berücksichtigung des Human- und natïrlichen Kapitals), jedoch durch stark negative „echte“ Ersparnisse von bis zu minus 15 Prozent des Sozialprodukts aus. Mit anderen Worten, der oftmals beschworene wirtschaftliche Erholungsprozeß Sub-Sahara-Afrikas ab Mitte der 80er Jahre ist zu einem Großteil einer unnachhaltigen Ausbeutung natülicher Ressourcen geschuldet.
Problematisch an Hamiltons Vorgehensweise ist jedoch seine theoretische Basis für die monetäre Berechnung der Abschreibungen des natïrlichen Kapitals. Als Abschreibungssatz wird der gesamte monetäre Ertrag aus dem Abbau natürlicher Ressourcen benutzt (der Nettopreis), was übertrieben erscheint, da somit aus dem Abbau natürlicher Ressourcen kein nachhaltiges Einkommen erzielt werden könnte. Diese Problematik hat El Serafy bereits 1989 beleuchtet und plädiert für einen Abschreibungssatz, der den Marshallschen Nutzerkosten entspricht (5). Dabei wird die statische Lebenserwartung der Ressource und die soziale Diskontierungsrate berïcksichtigt. Je höher die Diskontierungsrate und die statische Lebenserwartung der Ressource, desto geringer ist logischerweise der Anteil des Erlöses, der in andere Kapitalgüter investiert werden muß, um einen nachhaltigen Einkommensstrom zu garantieren. Würde El Serafys Ansatz auf Hamiltons Berechnungen angewandt werden, ergäben sich sicherlich weniger dramatische Resultate.

Weiterhin problematisch an Hamiltons Berechnungen ist, daß eine ,echte“ Ersparnis zwar als Indikator für schwache Nachhaltigkeit, aber nicht für nachhaltige Entwicklung dienen kann. Im Brundtland-Bericht wird jedoch explizit betont, daß nachhaltige Entwicklung nicht nur intergenerationelle Gleichheit, sondern auch intragerationelle Gleichheit im Sinne von allgemeiner Grundbedürfnisbefriedigung bedeuten muß. Dieser Aspekt ist aber in der Debatte um nachhaltige Entwicklung in Industrieländern weitgehend in den Hintergrund getreten. In Entwicklungsländern, die durch verbreitete Armut und hohe Einkommensunterschiede gekennzeichnet sind, wird diese Komponente nachhaltiger Entwicklung jedoch sehr viel mehr in den Vordergrund gerïckt.

\section{Ein never Indikator}

Um dem Rechnung zu tragen, habe ich in einer kürzlich in der Schriftenreihe des IÖW veröffentlichten Studie einen Indikator für nachhaltige Entwicklung vorgestellt, der zwar auf dem Modell der echten Ersparnis beruht, aber zusätzlich Indikatoren für eine ungleiche Verteilung von ökonomischen Ressourcen in der gegenwärtigen Generation beinhaltet (6). Dabei habe ich auf einen Indikator für Einkommensungleichverteilung verzichtet und statt dessen den Zugang zu Grundausbildung, Trinkwasser und einen Subsistenzeinkommen als Indikato- 
ren für Grundbedürfnisbefriedigung benutzt. Nachhaltige Entwicklung wäre gegeben, wenn diese Grundbedürfnisse für die gesamte Bevölkerung erfüllt sind und zusätzlich die echte Ersparnis größergleich Null ist, d.h. der Wert des Gesamtkapitalstocks (Sach-, Human-, und natürliches Kapital) über die Zeit nicht abnimmt. Diesen Indikator habe ich ,nachhaltige Ersparnis" genannt. Die nachhaltige Ersparnis gibt also an, welcher Betrag nach Abzug der Kosten allgemeiner Grundbedürfnisbefriedigung und der Kompensation der Abschreibungen des Gesamtkapitalstocks für sonstige Investitionen verbleibt. Ich habe damit die nachhaltige Ersparnis für Indien und Malaysia in den Jahren 1973-93 berechnet. Dabei wurden die Kosten für eine allgemeine Befriedigung oben genannter Grundbedürfnisse, Abschreibungen des Humankapitals durch Umweltverschmutzung, sowie Abschreibungen der wichtigsten natürlichen Rohstoffe, Kosten der Bodenerosion, des Waldsterbens, des Verlustes an Feuchtgebieten und der Kohlendioxid-Emissionen berücksichtigt. Für die Abschreibungen des natürlichen Kapitals wurde El Serafys Berechnungsmethode benutzt.

\section{Zwei Länderbeispiele}

Die Ergebnisse zeigen für beide Länder eine erhebliche Kluft zwischen konventioneller volkswirtschaftlicher Ersparnis und nachhaltiger Ersparnis. Im Falle Malaysias ist dies primär auf die hohen Abschreibungswerte des natürlichen Kapitals zurückzuführen (insbesondere Kohlendioxid-Emissionen, Schwund an Rohölreserven und Bodenerosion). Dennoch ist die nachhaltige Ersparnis dort in allen Jahren positiv, mit einem seit Mitte der achtziger Jahre hohen Stand allgemeiner Grundbedürfnisbefriedigung: Malaysia kann daher durchaus als ein Land beschrieben werden, daß sich auf einem ,schwachen“ nachhaltigen Entwicklungspfad befindet. Im Falle Indiens fallen hingegen gerade die schlechten sozialen Bedingungen ins Gewicht. Während die „echte" Ersparnis hier noch positive Ergebnisse in allen Jahren liefert, ist die nachhaltige Ersparnis bis Mitte der achtziger Jahre negativ, mit bis zu minus $18 \%$ des BIP. Hierfür verantwortlich ist vor allem der hohe Anteil der Armutsbevölkerung, aber auch die unzureichende Trinkwasserversorgung. Bei den ökologischen Indikatoren schlägt insbesondere die Bodenerosion $\mathrm{zu}$ Buche. Im Vergleich zu den Grundbedürfniskomponenten spielen die ökologischen Indika- toren allerdings eine untergeordnete Rolle. Eine Verwirklichung nachhaltiger Entwicklung in Indien muß daher in erster Linie Bekämpfung der Armut in der gegenwärtigen Generation bedeuten. Dies gilt um so mehr, als ein Großteil der dortigen Umweltzerstörung armutsinduziert ist.

Obwohl die Berechnung der nachhaltigen Ersparnis sicherlich ein Schritt in die Richtung einer integrierten 0perationalisierung nachhaltiger Entwicklung ist, soll noch einmal verdeutlicht werden, daß die wesentliche Schwäche des Indikators in der modellhaft angenommenen Substituierbarkeit der Kapitalformen liegt. Eine Quantifizierung nachhaltiger Entwicklung, die den realen Gegebenheiten besser Rechnung tragen will, wird nicht umhin kommen, einen kritischen, nicht-substituierbaren natürlichen Kapitalstock zu identifizieren.

\section{Anmerkungen}

(1) Report of the Ad Hoc Open-ended Inter-Sessional Working Group of the Commission on Sustainable Development (New York, 24 February-7March 1997) Document E/CN.17/1997/13

(2) Pearce, D.W./Atkinson, G. (1993): Capital Theory and the measurement of sustainable development: an indicator of weak sustainability. In Ecological Economics Vol. 8:

S. $103-108$

(3) Pearce, D.W./Atkinson, G. (1995): Measuring Sustainable Development, in: Bromley, D.W.: The Handbook of Environmental Economics.

Cambridge MA, USA, Blackwell

(4) Hamilton, K. (1995): Genuine Savings in Developing Countries. World Bank ESD Discussion Paper, Washington D.C., USA

(5) El Serafy, S. (1989): The Proper Calculation of Income from Depletable Natural Resources. in : Ahmad, Y.C./El Serafy, S./Lutz, E.: Enviromental Accounting of Sustainable Development. World Bank, Washington D.C., USA

(6) Hoas, C. (1997): Indikatoren für nachhaltige Entwicklung. Die Reform der volkswirtschaftlichen Gesamtrechnung am Beispiel Malaysia und Indien. Schriftenreihe des IÖW 113/97, Berlin

\section{Der Autor}

Diplom-Volkswirt Christian Haas nimmt zur Zeit am Ausbildungskurs des deutschen Instituts für Entwicklungspolitik (DIE) teil. Die Studie des Autors zum Thema kann in der ausführlichen Fassung als Schriftenreihe des IÖW Nr. 113/97 beim Institut für ökolo. gische Wirtschaftsforschung zum Preis von DM 27,00 angefordert werden.

Kontakt: DIE, Hallerstr. 3, 10587 Berlin, Tel. 030/39073-0

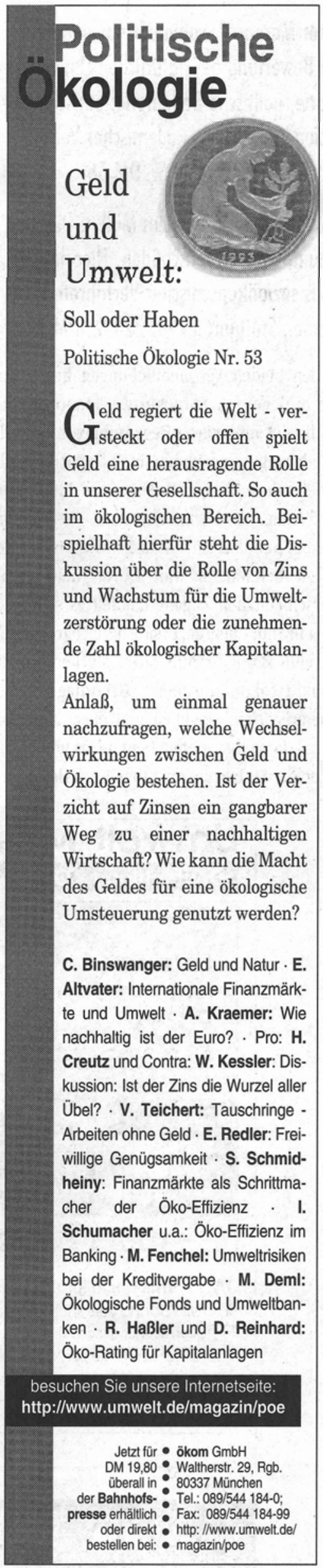


(c) 20I0 Authors; licensee IÖW and oekom verlag. This is an article distributed under the terms of the Creative Commons Attribution Non-Commercial No Derivates License (http://creativecommons.org/licenses/by-nc-nd/3.o/), which permits unrestricted use, distribution, and reproduction in any medium, provided the original work is properly cited. 